\title{
Research in engineering design: the role of mathematical theory and empirical evidence
}

\author{
Daniel D. Frey • Paulien M. Herder • Ype Wijnia • \\ Eswaran Subrahmanian · Konstantinos Katsikopoulos • \\ Richard de Neufville $\cdot$ Ken Oye $\cdot$ Don P. Clausing
}

Received: 18 January 2010/Accepted: 27 January 2010/Published online: 27 February 2010

(c) The Author(s) 2010. This article is published with open access at Springerlink.com

The editors of this journal have offered an opportunity to reply to Dr. Hazelrigg's letter in depth. Indeed, with its numerous points of critique of the paper "The Pugh Controlled Convergence method" (Frey et al. 2009) stated so strongly, the letter demands a detailed rebuttal. We provide a response to the specific points discussed in the letter as well as the broader issues raised. Writing on these topics has been an opportunity to explore some issues of interest to us, including the role of mathematical theory and empirical science in design research. To pursue this fully, additional authors participated to add more varied expertise on social sciences, preference measurement, and industry practices. We hope that our response will do more than defend the paper; we hope that it will also suggest some constructive paths forward in design research.

\section{The main point: interaction between analysis and synthesis}

To show that a wrong problem is being solved wrong in the sense that it is not the empirically given one - is the first ground for rejecting a theory: a matter of irrelevance. A second basis for rejection

D. D. Frey $(\bowtie) \cdot$ K. Katsikopoulos $\cdot$ R. de Neufville $\cdot$ K. Oye D. P. Clausing

Massachusetts Institute of Technology, Cambridge, MA, USA

e-mail: danfrey@mit.edu

P. M. Herder · Y. Wijnia

Delft University of Technology, Delft, The Netherlands

E. Subrahmanian

Center for Study of Science, Technology and Policy

(CSTEP), Bangalore, India would be to show that improper, inadequate, or oversimplifying assumptions have been made (Morgenstern 1972).

Research in engineering design, like all science, benefits from active critique based on both theory and empirical data. Hazelrigg's letter in effect asserts a veto power of his preferred mathematical theory over empirical evidence. For example, he writes "the reader of this or any other such paper should never merely assume that it is correct, but verify its validity through personal derivation". We agree that readers should never assume any particular publication is correct but disagree that personal derivation is an appropriate procedure in this context. If a paper presents data inconsistent with the hypotheses of a reader, a mathematical derivation will not give an adequate justification to ignore the data. A more appropriate procedure is to check the data for accuracy at their source, by replication of an experiment or by seeking data from other relevant records. If the data hold up to review, the deductive framework of the reader may need to be revised; for example, by changing its premises or by broadening the framework to incorporate more considerations. In the evaluation of Pugh Controlled Convergence, Hazelrigg's preferred mathematical framework suggests it will fail, but the evidence from practice indicates it does not. We submit that Hazelrigg's mathematical framework makes improper, inadequate, or oversimplifying assumptions.

The primary point of the paper "The Pugh Controlled Convergence method: model-based evaluation and implications for design theory" (Frey et al. 2009) is that decision-making (analysis) and alternative generation (synthesis) have significant interactions that should be modeled if one is to evaluate design methodologies. The paper discusses a documented case study (Khan and Smith 
1989), in which the chosen design concept was not among the initial set of design concepts being considered, but rather a new design that emerged after the engineers began the concept selection process. This is not an unusual occurrence in successful engineering design. We argue that such cases should not be ignored but instead should be considered as empirical evidence. In fact, the Pugh Controlled Convergence method provides specific mechanisms to encourage the development of new design concepts as evaluation of the extant concepts proceeds. This implies that, to assess such a design method, it is essential to consider the upside of generating additional design alternatives along with the potential downside of any simplifying assumptions. Hazelrigg's framework assumes a set of alternatives as input and assumes that success in design is primarily a function of how well decisions are made among this fixed set. This is the central issue in the paper that leaving out generation of alternatives from a framework for design scholarship severely limits its descriptive and prescriptive value. This was not mentioned anywhere in Hazelrigg's letter. The Decision Based Design framework as described by Hazelrigg (1998) excludes generation of alternatives and therefore fails to address the "empirically given" problem of engineering design, to borrow the terminology of Morgenstern (1972).

The claims made in Frey et al. (2009) are open to empirical testing by the design research community. One of our claims is that teams using Pugh Controlled Convergence in the concept design phase will, on average, produce better designs than teams implementing the suggested remedy in Saari (2006). We encourage other researchers to also run experiments and to study historical records to evaluate this and other claims. By contrast, Hazelrigg explicitly discourages empirical work in design. His letter suggests that the impact of design methods on design outcomes is "unknowable." We assert that this notion is mistaken, and it would be an impediment to progress in engineering design if the community were to accept it.

\section{Professional practice}

[C]ontroversies about the foundations... reflect themselves to some extent in everyday practice, but not nearly so catastrophically as one might imagine (Savage 1954).

Engineering has been an important part of human culture for a long time, and the engineering profession has therefore accumulated a great deal of explicit and tacit knowledge about what works and what does not. Hazelrigg writes that "the authors argue that the extant methods, Pugh included, obviously must have value since engineering has done so well ...." To be precise, Frey et al. (2009) do not claim it is "obvious", but we clarify here that there is a connection between the value of methods and their frequency in use.

The set of practices currently in wide use has coevolved with the engineering profession. In somewhat Darwinian fashion, firms that adopted relatively weaker practices tended to suffer. If people observed a connection between weak engineering practice and poor results, they sometimes adjusted by copying the practices of firms with better results. If they failed to make appropriate changes, they more quickly went out of business, and so weak practices tended not to be copied. Even though engineering can always be improved, claims of "serious flaws" or "invalidity" of any engineering method in wide use should be viewed cautiously. The burden of proof therefore lies primarily upon the ones claiming the existence and importance of the flaws. They must provide specific evidence of the failures they claim actually occurring in professional practice (toy problems are interesting but insufficient). They must provide credible data on the frequency of occurrence and severity of the consequences. When remedies are suggested, they must demonstrate that the changes in the design methods do not cause damaging side effects that outweigh their benefits.

Let us consider one specific domain of engineering as a case in point - design and manufacture of jet engines. In the period from 1960 to 2000, engineers evolved the design of gas turbine engines, resulting in improving thrust by a factor of five, improving thrust-to-weight ratio and thrust-specific fuel consumption by a factor of two, reducing noise by about $20 \mathrm{~dB}$, increasing engine life-on-wing by about an order of magnitude, and increasing reliability (based on frequency of in-flight shutdowns) by two orders of magnitude (Koff 2004; Ballal and Zelina 2004). These successes of the past were obtained by the extant methods used over time by General Electric, Pratt and Whitney, Rolls Royce, smaller competitors, and a whole system of suppliers to this industry. The design methods were not static but improved along with the engines themselves. For example, statistical methods for quality engineering were developed, widely disseminated within companies, and spread between companies over time. Improvements in design methods are still to be discovered and implemented. It is possible that more rigorous decision analysis will be part of that improvement, but calls for wide-scale rejection of current, successful industry practices are counterproductive.

\section{Voting and social choice}

The possibility of constructive ... social choice ... turns on broadening the informational basis of such decisions (Sen 1998). 
"The Pugh Controlled Convergence method" (Frey et al. 2009) states that "there is no voting in Pugh method." Hazelrigg counters that voting is used both "to obtain consensus on the relative merits" and "to aggregate the symbols", and thus the Pugh method is invalidated based on Arrow's Impossibility Theorem. It seems we must disagree on either what the word "voting" means or on what Pugh method entails. We tried to understand this dispute over the concept of "voting" by showing a sample of political scientists and sociologists a video of engineers using the Pugh method (facilitated by Stuart Pugh) and asked them whether they thought the engineers voted to obtain consensus on the relative merits. The majority of scholars we polled answered "no" and usually made stronger statement like "definitely not." We therefore believe that people who understand voting and have accurate knowledge of the Pugh method usually agree that there is no voting used to assign symbols to the matrix. We encourage others to try similar tests and to determine for themselves if voting is essential for a group to decide whether an engineering alternative is better or worse than another on some specific criterion.

Voting is also not needed "to aggregate the symbols + , -, S." Often, sums are presented at the bottom of the matrix, but purely as a guide for discussion. The sums have no power to make a decision to eliminate or retain an option. In the Pugh process, the lead engineer on the project is not absolved from responsibility for these decisions. Pugh laid this out explicitly in his books and papers. To create a mathematical model of the Pugh process, we chose an algorithm for deciding which concepts to eliminate (an algorithm similar to patterns in current practice), but we also explained that the actual Pugh method is not based on any automated decision-making algorithm.

It is useful to consider why this disagreement about the term "voting" came about, and what it means for design research. The disagreement is understandable, because the Pugh method does involve a facilitator asking participants for their opinions, and one can interpret a request for an opinion as an opportunity to vote. But the purpose of the request, in this case, is not to tally, but to give each participant a change to explain their reasons, to bring new information to light, and potentially to change the opinions of other participants.

The standard use of the term "voting" includes some formal process of counting the votes and comparing them to a predefined standard for passage of a measure. The Pugh process does not normally have this feature. In the Pugh method, the focus is not on outnumbering the dissenters, but on giving them an opportunity to persuade others. This is the essence of consensus decision-making methods such as the Polder model. The political and social scientists to whom we showed the videos generally make a distinction between a process of building consensus and a process for taking a vote.
We emphasize that claiming there is no voting in the Pugh method is not the same as asserting that there are no significant challenges in social choice relevant to engineering. For example, if even a single engineer on the team was intent on advancing a favorite design concept and that engineer would not listen to and learn from others, then the Pugh method would run into serious difficulty. For example, the favored alternative could not earn less than an ' $S$ ' rating in any criterion if the proponent would not concede to any weaknesses of his favorite alternative. This would greatly reduce the value of the information displayed in the matrix, and convergence would be difficult to achieve. The procedure proposed by Saari (2006) also offers no useful remedy to the problem of people who are wrong and who are unwilling to learn. A carefully crafted voting procedure applied to an uninformed group is useless. The Pugh method does not have any absolute remedy either, but it does at least prompt the group to discuss all the alternatives and their merits in every criterion. The Pugh method begins to address an authentic problem. The engineering profession, by its nature, requires diverse disciplinary representation on teams so that listening to others and sharing knowledge are essential to good design (Konda et al. 1992). The problems caused by stubborn people, selfsealed against learning, are real and serious. By comparison, the concerns posed by Arrow's theorem are minor.

\section{On the role of shortcuts in engineering}

\section{Models have to be fashioned with an eye to practical computability, no matter how severe the approxima- tions and simplifications that are thereby imposed on them (Simon 1978).}

Engineers sometimes choose one design method over an alternative because it is easier to learn or easier to apply. Hazelrigg implies that engineers who do this "do not care about the outcome" and claims that "shortcuts really do not work." Our paper makes a clear statement on this by describing a specific scenario in which a task could be accomplished using the Pugh method in one 8-h workday. We show that employing Saari's suggestion to do the same task would require the same team of engineers to spend almost 2 weeks of all-day meetings to make the same decision. Hazelrigg does not challenge our time estimates, he simply insists, inexplicably, that any argument regarding the effort required is "utterly specious".

Engineering design has to be carried out under deadline and budget constraints, and engineers must decide how to make decisions based partly on how much time and energy the available methods require. Engineers must seek the most efficient approaches (which can be characterized 
loosely as "shortcuts") to complete their work under realistic constraints, and these are particularly valued in the early stages of design. As an example, gas turbine engine designers will typically specify a simplified analysis (onedimensional, steady-state) for an early stage of engine design. More complex analyses that deal with the component behaviors including three-dimensional and unsteady effects will not be attempted until later in the design process. As Frey et al. (2009) suggest, a similar pattern can probably be recommended for decision-analysis with simple procedures in the concept design phase (e.g., avoiding weighting of criteria), and more elaborate trade-off studies deferred until later in the design process.

We assert that a major factor in an engineer's professional success is the ability to simplify a task when possible without compromising the outcome in terms of the project goals. This is a key ability we emphasize in engineering schools. We want engineers to make simplifying assumptions when appropriate. We are delighted when students reframe a difficult analysis so that it becomes more clear and is still basically correct. This is not evidence of laziness, it is a demonstration of engineering skill. In many cases, the simplification is not only a key to a faster estimate, but also a key to insight. The value of simplification can be observed both in analysis of physical phenomena and in decision analysis.

\section{On the risk of poor decisions}

No model, or results of an analysis, ever makes a decision. The decision makers ... must make the decision (Keeney 2009).

Hazelrigg claims the Pugh method can potentially select the worst option among the given alternatives. According to our model, it is highly unlikely that the Pugh method will lead to selecting the worst option. Hazelrigg makes a related claim that "as the number of attributes across which alternative designs are evaluated increases and as the number of design alternatives increases, the likelihood of obtaining quite poor results becomes quite significant." This is just the sort of statement that our model is intended to evaluate. The results of our model are not consistent with Hazelrigg's statement about scale-up and the results of the Pugh method. It is true that the probability of choosing the single "best" design decreases with the number of alternatives in the initial set. But, given reasonable assumptions, the engineering results remain good according to our model. That is because adding more alternatives tends to increase the number of strong alternatives that are comparably good to the "best" concept. This is a phenomenon that is also observed in engineering practice. As jet engines have evolved, competitors have pursued fairly distinct designs. At present, General Electric, Pratt and Whitney, and Rolls Royce offer different engine designs; and all three can be available for the same airframe. The three distinct designs are often comparable in overall merit considering technical, economic, and logistical factors.

The point is that as the number of design options increases, a significant number of options emerge that are comparable in their merits, which is consistent with our model's behavior. In these circumstances, choosing a design concept may actually be easier-any one of the good concepts could be successful. On the specific question of whether the Pugh method frequently fails badly or not, we seem to be at a standoff, in which Saari's model supports one conclusion and our model supports a different conclusion. To make a more definitive assessment, we suggest that trials should be conducted by teams of experienced engineers working with realistic design alternatives evaluated against authentic engineering criteria. If the engineers frequently choose poorly, we would consider that to be worthy of serious concern in the design theory community. Currently, however, we do not consider Saari's theorem by itself to be a sufficient motivation for widescale change in current design methods.

Hazelrigg states "the authors argue that they remove design alternatives from consideration only when they are dominated by other alternatives, inferring that this provides stability in convergence to an optimal design." This is not an accurate description of our paper which demonstrated that the model of a Pugh procedure, in the preponderance of instances, provides convergence to a competitive design concept. On a related note, Hazelrigg states "Saari shows cases where such actions can completely flip the order of the remaining alternatives." To be precise, these things Saari shows are not "cases" in the sense that most people use the term. Neither Saari nor Hazelrigg presents any evidence that these events ever actually happened, either through their own casework or by citing any previously published accounts. Saari shows that you can devise fictional instances wherein the reversals could theoretically occur. Our models suggest that consequences of Saari's concerns are mild, and the evidence from engineering practice is consistent with that position.

Better decision-making methods can and should be devised. But Hazelrigg's framework appears to transfer complex technical decisions away from the judgment of experienced professionals and leave them up to explicit computational procedures. This could degrade the quality of decisions, especially when tacit knowledge plays a role in the expert's judgements. Because of these considerations, we submit that any new procedures for decision-making should 
be evaluated against some of the best human practitioners before they are placed in wide use.

\section{On the standing of counterexamples as evidence}

Not everything that looks like a fallacy... is one (Gigerenzer et al. 2008).

Hazelrigg makes an interesting analogy. On the one hand, there is a design method that could, in some instance, lead to a suboptimal decision. We are asked to compare this with a proposed operation for adding two or more numbers, viz., asserting that their sum will always be 100 . Sometimes, this operation is right, most of the time it is wrong. We think the point he is trying to make has to do with the standing of counterexamples as evidence. In arithmetic, it is a serious concern if a single example shows that your procedure can give a wrong result. In arithmetic, there are alternative procedures that give correct results over the whole domain, so it is better to use those for performing addition. By contrast, there are no methods that give "correct results" over the "whole domain" of engineering design (if we pick any reasonable definition of its domain). As our paper emphasizes, generating design alternatives would surely have to be part of the engineering design domain. If design concept generation is admitted as part of the domain, we can not even say what the term "correct results" means, and we have to be satisfied with pursuing "better results." The point is that arithmetic is a poor analogy regarding the assessment of evidence and consideration of counterexamples in engineering design.

Regarding potential for error arising from a design method, a more apt analogy is the human system for visual perception. People are adept at assessing evidence in the form of light reflecting from surfaces, using this input to perceive position, size, orientation, and texture, and inferring facts about the objects and scenes in their environment. On the other hand, it is easy to construct examples of difficulties in human visual perception; we often call these examples "optical illusions." When people are presented with such illusions, they may find them disconcerting, but they generally do not stop trusting their vision completely. When people discover a real and serious problem with their vision (e.g., astigmatism), they will seek a means to correct it if a reliable means is available. But people do not take any countermeasures against common optical illusions, because in realistic circumstances, they do not appear with any significant frequency nor do they cause serious negative consequences. We propose that the concerns Hazelrigg (1998), Franssen (2005), and Saari (2006) describe are similar to optical illusions. They are interesting to know about, and they might even be useful tools for exploring the mechanisms of human decision-making, and it would be interesting to see if these "cognitive illusions" can explain some of the specific decisions from the jet engine industry as described by Koff (2004). But, by themselves, artificial examples do not compel anyone to hastily change their current engineering design methods.

Along a similar line of reasoning, seeking an exceptional case with a bad outcome, Hazelrigg asserts that if you choose a design with the best values on several different attributes "it is entirely possible that this is the worst design." Frey et al. (2009) does not dispute this as a possibility, but we clarify now that it is generally not a realistic concern in engineering practice. For example, in the selection of a jet engine design, criteria considered typically include weight (for a given thrust mission), thrust-specific fuel consumption, manufacturability, direct operating costs, and operability (e.g., stall avoidance and frequency of in-flight shutdown). Hazelrigg is asking the reader to worry about the following possibility - you happen to have an engine design that is best among a set of alternatives in all these criteria, i.e., it is the lightest, uses the least fuel, it is the easiest to manufacture, it operates most reliably, costs the least to make and to operate, but this same engine is nevertheless the worst option because somehow that combination of properties will be bad for the airline. How exactly might this occur unless a major criterion is missing? Are there any welldocumented examples of experienced engineers making poor decisions that were caused by these multi-attribute interactions? Do these occur with high frequency or severity? These questions should be answered before theorists recommend changes in engineering practice.

\section{On the role of decision analysis and the nature of preferences}

\section{.. the goal of maximizing utilities seems to be} undermined by this very goal (Schooler et al. 2003).

Hazelrigg writes, "Preferences are in the head of the decision maker and, thus, are known with certainty." We think that in light of previous papers (e.g., Hazelrigg 1996), it would be more appropriate for him to say "in my mathematical model of decision making, I assume preferences are known with certainty." The term as written in the letter encourages the reader to suppose it is a statement about reality, in particular because Hazelrigg refers to the "head" of a decision-maker which is a vivid image to suggest that the decision-maker is a human. So, we analyze the statement as if it was an empirical claim about humans. Science supports the contention that "preferences are in the head of the decision-maker." Kable and Glimcher (2007) write, "Revealed preference theories in economics posit that 
decision makers behave as though different options have different subjective values. Here we have shown that neural activity in several brain regions ... tracks the revealed subjective rewards. ... These results indicate that choosing ... involves, at a physical level, comparing neurally encoded subjective values." We conclude that science is emerging that is beginning to establish how and where preferences are in the head of the decision-maker, that is, how they are encoded by the states of neurons. Now, consider the second part of Hazelrigg's claim - that preferences are "known with certainty." We assume here that Hazelrigg is referring to the decision-maker's knowledge of their own preferences. Humans can be said to "know" their preferences to some degree because they can access their memories, imagine their future behaviors, and try to predict their own actions. But current science strongly contradicts the view that humans can assess "with certainty" their own preferences if the term "preference" is defined in a way that allows for empirical assessment (see for a review Schooler et al. 2003). Research provides strong evidence that humans assess their own preferences using the same procedures and even the same brain regions that enable them to think about other people's thoughts (e.g., Lombardo et al. 2009; Vogeley et al. 2001; Carruthers 2009). People have considerable conscious access to their own mental states, but these assessments are not made with certainty. It is possible to assert that whatever people report as their preference at a particular moment is defined to be their preference, but if preferences can change over time and can be distorted by the introspective process itself, as research shows, then this claim is merely circular and not meaningful as empirical science. We submit that Hazelrigg's statement, if it has meaning outside a purely mathematical construct, is known by scientists to be false. On a related topic, for details on Hazelrigg's mischaracterization of Arrow's theorem, see, for example, Keeney (2009). To decide for your self if what we are saying is true, we encourage members of the engineering design community to engage with cognitive scientists, psychologists, behavioral economists, and other scholars who are interested in decision-making as an empirical science.

\section{On the example presented in the appendix}

It is exceptional that one should be able to acquire the understanding of a process without having previously acquired a deep familiarity with running it, with using it, before one has assimilated it in an instinctive and empirical way... [John von Neumann, as quoted in Newman (1956)]

The appendix of the letter includes a table that is intended to serve as a "Pugh example illustrating misbehavior." This table is in no reasonable way an example of the use of Pugh methods. It is deficient in many dimensions especially in authenticity, accuracy in representing the process, and legitimacy in representing the outcomes.

Our paper (Frey et al. 2009) includes discussion of authentic examples of Pugh method and citations of papers presenting further details. These examples present tables including alternatives and the symbols assigned to them. Hazelrigg's table also lays out alternatives and symbols, but the similarity ends there. In the examples we cite, the symbols in the matrix represent work done by engineers assessing the relative merits of actual engineering design concepts. By contrast, the table presented by Hazelrigg has no correspondence to specific real-world events. Readers should be aware of this distinction.

On a related issue, in the examples of Pugh method cited in Frey et al. (2009), when a design alternative is eliminated or a single design is finally chosen, that is a documentation of what the people involved decided based on the evidence and their discussions thereof. By contrast, Hazelrigg's example presents what he calls the "Pugh preferred design." We do not believe there is such a thing. We emphasize that there is no preference for a design alternative implied by the tallies at the bottom of a Pugh matrix. These are merely summaries or descriptive statistics that have no force to compel the decision-makers. Hazelrigg asserts that Pugh methods lead to the choice $\mathrm{X}_{1} \mathrm{Y}_{1} \mathrm{Z}_{1}$. The models in our paper can be used to assess the frequency that teams choose $X_{1} Y_{1} Z_{1}$, and we conclude that this choice is very rare. But this requires a number of assumptions about how to represent the scenario. The critical question is how experienced practitioners would represent a scenario like this, and what decision they would actually make using the Pugh method. We hope this experiment will be conducted, preferably by multiple independent investigators.

Most importantly, in published examples of people actually using the Pugh method, the chosen concept is rarely among the alternatives initially considered. The central point of our paper is that Pugh method explicitly encourages people to generate additional alternatives. If an actual group of people were faced with a scenario similar to the one in this table, they would be encouraged by the Pugh method to develop additional alternatives. In particular, especially since customers are listed in the rows, the Pugh method would encourage marketing experts to be involved in the decision. Therefore, the range of alternatives to be considered ought to include approaches to segmenting the market as well as approaches to finding individual, new designs that all three customers will want to purchase. We strongly encourage researchers to develop more authentic examples of such tough situations and have teams of 
engineering and marketing professionals trained in Pugh Controlled Convergence work on the design challenges. Hazelrigg seems to imply that his framework would result in better outcomes than Pugh methods. We are interested to know what will happen in realistic head-to-head tests.

\section{Conclusion}

\section{Intellectual honesty does not consist in trying to entrench, or establish one's position ... it ... consists rather in specifying precisely the conditions under which one is willing to give up one's position (La- katos 1970).}

This document has been written as a defense of the data, methods, and claims in "The Pugh Controlled Convergence method: model-based evaluation and implications for design theory" (Frey et al. 2009). The fact that we are defending that position, however, does not imply the position will not change in the face of new evidence. The philosopher of science Imre Lakatos introduced an important distinction between progressive and degenerating research programs (1973). He posited that a progressive program is marked by the discovery of novel facts, development of new experimental techniques, and more precise predictions; a degenerating research program is marked by lack of discovery of novel facts and growth of a protective belt that seeks to insulate it from empirical falsification. We seek to be part of a progressive research program and hope to evolve that program in concert with other researchers in engineering design.

\begin{abstract}
Acknowledgments The advice of many colleagues has been helpful in writing this paper. In particular, Edward Greitzer helped with our discussion of jet engines and jet engine design. Financial support from the National Science Foundation (grant \#0448972) is gratefully acknowledged.
\end{abstract}

Open Access This article is distributed under the terms of the Creative Commons Attribution Noncommercial License which permits any noncommercial use, distribution, and reproduction in any medium, provided the original author(s) and source are credited.

\section{References}

Ballal DR, Zelina J (2004) Progress in aeroengine technology (19392003). AIAA J Aircr 41:43-50

Carruthers P (2009) How we know our own minds: the relationship between mindreading and metacognition. Behav Brain Sci 32(2): $121-138$

Franssen M (2005) Arrow's theorem, multi-criteria decision problems and multi-attribute preferences in engineering design. Res Eng Des 1:42-56
Frey DD, Herder PM, Wijnia Y, Subramanian E, Katsikopoulos K, Clausing DP (2009) The Pugh controlled convergence method: model-based evaluation and implications for design theory. Res Eng Des 20(1):41-50

Hazelrigg GA (1996) Systems engineering: an approach to information-based design. Prentice Hall

Hazelrigg GA (1998) A framework for decision-based engineering design. ASME J Mech Des 120:653-658

Gigerenzer G, Hertwig R, Hoffrage U, Sedlmeier P (2008) Cognitive illusions reconsidered. In: Plott CR, Smith VL (eds) Handbook of experimental economics results. Elsevier, Amsterdam

Kable JW, Glimcher PW (2007) The neural correlates of subjective value during intertemporal choice. Nat Neurosci 10(12):16251633

Keeney R (2009) The foundations of collaborative group decisions. Int J Collab Eng 1(1):4-18

Khan M, Smith DG (1989) Overcoming conceptual barriers-by systematic design. Proceedings of the Institute of Mechanical Engineers ICED, Harrogate

Koff BL (2004) Gas turbine technology evolution: a designer's perspective. J Prop Power 20(4):577-595

Konda SL, Monarch I, Sargent P, Subrahmanian E (1992) Shared memory: a unifying theme for design research and practice. Res Eng Des, Special Issue Des Human Context, H. Petroski (Ed), 4(1):23-42

Lakatos I (1970) "Falsification and the methodology of scientific research programs", from Criticism and the growth of knowledge, in Schick: Readings in the Philosophy of Science

Lakatos I (1973) "Science and pseudoscience", Broadcast June 30, 1973, as Programme 11 of The Open University Arts Course A303, "Problems in Philosophy", by the BBC. Pub. as Philosophy in the Open, ed. by Godfrey Vesey, and as the Introduction to The Methodology of Scientific Research Programmes, ed. by John Worrall and Gregory Currie

Lombardo MV, Chakrabartia B, Baron-Cohen S (2009) What neuroimaging and perceptions of self-other similarity can tell us about the mechanism underlying mentalizing. Behav Brain Sci 32:152-153

Morgenstern O (1972) Thirteen points in contemporary economic theory: an interpretation. J Econ Lit 10(4):1163-1189

Newman JR (1956) "The mathematician" in the world of mathematics. Dover Publications, Mineola

Saari DG, Sieberg KK (2006) Are partwise comparisons reliable? Res Eng Des 15:62-71

Savage L (1954) The foundations of statistics. Dover Publications Inc., New York

Schooler JW, Ariely D, Loewenstein G (2003) The pursuit of happiness can be self-defeating. In: Brocas I, Carillo J (eds) The psychology of economic decisions. Oxford University Press, Oxford

Sen A (1998) The possibility of social choice, Prize Lecture: The Sveriges Riksbank Prize in Economic Sciences in Memory of Alfred Nobel, Trinity College, Cambridge, UK. http://www. nobelprize.org/nobel_prizes/economics/laureates/1998/sen-lecture. pdf

Simon H (1978) Rational decision-making in business organizations. Prize Lecture: The Sveriges Riksbank Prize in Economic Sciences in Memory of Alfred Nobel, Carnegie-Mellon University, Pittsburgh, Pennsylvania, USA. http://www.nobelprize. org/nobel_prizes/economics/laureates/1978/simon-lecture.pdf

Vogeley K, Bussfeld P, Newen A, Herrmann S, Happe F, Falkai P et al. (2001) Mind reading: neural mechanisms of theory of mind and self-perspective. Neuroimage 14(1 Pt 1):170-181 